\title{
Estudio comparativo de reconstrucción de ligamento cruzado anterior entre las técnicas de Un Haz y U-Dos
}

\author{
Comparative study of anterior cruciate ligament reconstruction \\ between the One Band and U-Two techniques
}

\author{
Berumen-Nafarrate E,* Carmona-González J, ${ }^{\ddagger}$ Tonche-Ramos JJ, ${ }^{\ddagger}$ Carmona-Máynez O,* \\ Aguirre-Madrid A,* Moreno-Brito V, ${ }^{\ddagger}$ Leal-Berumen $\mathrm{I}^{\ddagger}$
}

Hospital Christus Muguerza del Parque, Chihuahua, Chihuahua.

RESUMEN. Introducción: Las lesiones del LCA se encuentran dentro de las lesiones ligamentarias más frecuentes. Esto ha llevado al desarrollo de diferentes técnicas quirúrgicas para recuperar la estabilidad de la rodilla. Material y métodos: Estudio clínico, aleatorizado, ciego comparando técnicas de reconstrucción de LCA con Un Haz y «U-Dos» mediante escalas clínicas y artrometría con KT-1000 y Pivot Shift Meter. Resultados: De 36 pacientes, 52.94\% fue sorteado para la técnica de Un Haz y 47.06\% para «U-Dos». En 11.76\% de los pacientes se presentó un desplazamiento mayor a $11 \mathrm{~mm}$ en la rodilla afectada con KT-1000. En 8.82\% se mostró un desplazamiento anterior igual en ambas rodillas. Un paciente presentó un desplazamiento anterior mayor posterior a la cirugía de reconstrucción con la técnica de Un Haz, pero con mejoría en las escalas clínicas. En las mediciones de Pivot Shift se encontró una diferencia en el desplazamiento mayor a los 4,000 m/s en el eje de las X tras la técnica de Un Haz en comparación con la rodilla sana calculando integrales, mientras que con U-Dos la diferencia fue menor de $600 \mathrm{~m} / \mathrm{s}$. En el eje de «Y» se encontró una diferencia $>3,500 \mathrm{~m} / \mathrm{s}$ entre la pierna sana y la lesionada, la cual disminuye a menos de 2,000 m/s tras la reconstrucción mediante técnica de U-Dos. Conclusiones: A lo largo del estudio se han encontrado diferencias en la evolución de los pacientes que indican que la reparación mediante la técnica de U-Dos tiene mejores resultados al lograr recuperar la estabilidad anteroposterior y rotacional del miembro afectado.

Palabras clave: Ligamento cruzado anterior, reconstrucción, estabilidad, acelerómetro.
ABSTRACT. Introduction: ACL injuries are found within the most common ligament injuries. This has led to the development of different surgical techniques to restore knee stability. Material y methods: Clinical study, randomized, blind comparing ACL reconstruction techniques with One Band and «U-Two» using clinical scales and arthrometria with KT-1000 and Pivot Shift Meter. Results: Of 36 patients, 52.94\% was drawn for the One Band technique and $47.06 \%$ for «U-Two». 11.76\% of patients had a displacement greater than $11 \mathrm{~mm}$ in the affected knee with KT-1000. 8.82\% showed an equal anterior displacement on both knees. One patient had a major previous displacement after reconstruction surgery with the one band technique, but with improvement on clinical scales. Pivot Shift measurements found a difference in displacement greater than $4,000 \mathrm{~m} / \mathrm{s}$ on the $\mathrm{X}$ axis following the One Band technique compared to the healthy knee calculating integrals, while with U-Two the difference was less than $600 \mathrm{~m} / \mathrm{s}$. On the $\mathrm{Y}$ axis a difference of $>3,500 \mathrm{~m} / \mathrm{s}$ was found between the healthy and the injured leg, which decreases to less than 2,000 m/s after reconstruction using U-Two technique. Conclusions: Differences have been found throughout the study in patient evolution, indicating that repair using the U-Dos technique has better results in recovering the anteroposterior and rotational stability of the affected limb.

Keywords: Anterior cruciate ligament, reconstruction, stability, accelerometer.

\section{Nivel de evidencia: II}

\footnotetext{
* Ortopedia y Traumatología, Hospital Christus Muguerza del Parque. Chihuahua, Chihuahua.

‡ Facultad de Medicina y Ciencias Biomédicas, Universidad Autónoma de Chihuahua.

Dirección para correspondencia:

Irene Leal-Berumen

Facultad de Medicina y Ciencias Biomédicas, Universidad Autónoma de Chihuahua, Chihuahua, México. Tel: (614) 4103320

E-mail: ileal@uach.mx
} 


\section{Introducción}

El ligamento cruzado anterior (LCA) es un ligamento intraarticular que consiste en dos haces, el anteromedial (AM) y posterolateral (PL). Ambos trabajan de forma sinérgica para controlar y limitar la traslación y rotación axial de la rodilla. Debido a la alta incidencia de las lesiones del LCA tanto en el ámbito deportivo como incidental, su reconstrucción es un procedimiento relativamente común dentro de la práctica diaria de los cirujanos ortopedistas. ${ }^{1,2}$

La técnica de Un Haz se realiza produciendo un solo túnel femoral y uno tibial; ésta se enfoca primariamente en la reconstrucción del haz anteromedial mediante la colocación de un aloinjerto. Se han reportado estudios que han demostrado que esta técnica es insuficiente para controlar el movimiento rotacional y el valgo forzado que simula la maniobra de pivot shift. ${ }^{3}$ De acuerdo a las observaciones realizadas por Tashman y colaboradores (2004), la reconstrucción del LCA por medio de la técnica de Un Haz restablece exitosamente el desplazamiento anterior/posterior, pero falla en restablecer la estabilidad rotacional normal. ${ }^{4}$

En la técnica de doble haz, los haces AM y PL son reconstruidos con aloinjertos por separado para simular mejor la anatomía normal del LCA, en ésta se observa un mayor control rotacional y funcionalidad total de la rodilla. Sin embargo, entre las desventajas se considera compleja, consumidora de tiempo, de mayor costo y con mayor grado de dificultad en comparación con la de un haz. ${ }^{5,6}$ Meredick y colegas (2008) mostraron a través de ensayos controlados aleatorizados que no había diferencias clínicas significantes entre la técnica de Un Haz y la de doble haz utilizando el KT-1000 y la maniobra Pivot Shift para valorar la estabilidad anteroposterior como rotacional. ${ }^{7}$

Recientemente ha sido descrita una modificación de la técnica de doble haz llamada «U-DOS», en la cual se utiliza un aloinjerto que se espera solucione los problemas de inestabilidad y aumento de rotación externa, evitando la degeneración temprana de la articulación. ${ }^{8}$ Brevemente, esta técnica se realiza por medio de artroscopía, se colocan dos clavos guía en el fémur y tibia para después perforar los túneles con la broca. Por dichos túneles se pasan los clavos guía que ayudan a trasladar las suturas que se encuentran unidas a los extremos del aloinjerto, en sentido distal a proximal, quedando los extremos dentro de los túneles femorales. Se fija el injerto en su haz anterior a $60^{\circ}$ de flexión y el haz posterior a $110^{\circ}$ de flexión, esto por medio de dos tornillos biocompuestos en ambos túneles femorales, mientras la fijación a nivel tibial se dará por el puente óseo, sobre el cual se coloca la «U» del aloinjerto. Dentro de sus ventajas se encuentran: restablecer una posición anatómica de los haces, utilizar sólo dos tornillos, reducir costos en comparación con la técnica de doble haz convencional, además de representar menos tiempo intraoperatorio. ${ }^{8,9} \mathrm{El}$ objetivo del presente trabajo es comparar las técnicas de Un Haz y UDos para la reparación del LCA utilizando el KT-1000 como estándar de oro, acelerómetros para la prueba de pivote y escalas funcionales de Tegner Lysholm e IKDC valorando la evolución clínica del paciente tras un seguimiento a dos y tres meses de la cirugía.

\section{Material y métodos}

\section{Escalas utilizadas}

Tegner Lysholm: La escala de Tegner Lysholm está diseñada para informar sobre la posibilidad de realizar actividades cotidianas mediante una serie de preguntas que involucran signos y síntomas como la cojera, dolor, inflamación, sensación de bloqueo y de inestabilidad; así como actividades cotidianas que incluyen subir escaleras y ponerse en cuclillas.

Cuestionario IKDC (International Knee Documentation Committee): El cuestionario de IKDC es una escala subjetiva que provee a los pacientes de una calificación funcional general. El mismo abarca tres categorías. Sintomatología (dolor, rigidez, inflamación y estabilidad). Actividades físicas cotidianas como el subir y bajar escaleras, levantarse de una silla, ponerse en cuclillas y saltar. Finalmente la función de la rodilla previo a su lesión y posterior a la misma. Tras realizar el cuestionario se obtiene un puntaje de 0-100 reportando un mejor funcionamiento entre mayor sea la calificación obtenida.

\section{Equipo utilizado}

KT-1000 Medmetric Knee Ligament Arthrometer. Es la prueba considerada «estándar de oro»; en ésta se utiliza un artrómetro, el cual se coloca y se ajusta sobre la pierna a revisar y en reposo se evalúa el desplazamiento anterior de la tibia sobre el fémur midiendo la maniobra de «cajón» en milímetros. Si el resultado fuera mayor a $11 \mathrm{~mm}$ en una sola pierna o una diferencia mayor de $3 \mathrm{~mm}$ entre ambas rodillas se considera positivo para la lesión del LCA.

Pivot Shift Meter: Dispositivo que consiste en un par de acelerómetros y un giroscopio unidos por bandas elásticas, las cuales se deben ajustar por encima de la rótula y sobre la tuberosidad tibial. Una vez ajustado el equipo se abre el programa en la computadora para registrar el movimiento de los acelerómetros durante la realización de la maniobra.

Se trata de un estudio clínico prospectivo y aleatorizado, aprobado por el Comité de Ética del Hospital Christus Muguerza del Parque, en el cual se incluyeron a 36 pacientes en un rango de edad de 12 a 60 años de edad que accedieron a formar parte del protocolo y firmaron un consentimiento informado. El promedio de edades fue de 33.4 años, abarcando desde los 14 hasta 60 años. La población constó de 73.53\% de hombres y $26.47 \%$ de mujeres; todos con diagnóstico comprobado de lesión del LCA mediante pruebas de gabinete y maniobras clínicas. Todos ellos fueron evaluados utilizando la escala de Tegner Lysholm y el cuestionario del IKDC, así como el KT-1000 y el Pivot Shift Meter con la finalidad de evaluar la evolución postoperatoria de los pacientes con las diferentes técnicas de reconstrucción de LCA. 
Se excluyeron a los pacientes que no accedieron a firmar el consentimiento informado, aquéllos intervenidos quirúrgicamente previo al estudio por lesión de LCA y sujetos con enfermedades crónico-degenerativas de la rodilla afectada o que en su defecto fueran portadores de prótesis de rodilla.

La valoración del paciente inició al realizar ambos cuestionarios previamente mencionados para obtener los puntajes preoperatorios; después se procedió a la evaluación clínica mientras el paciente se encontraba bajo el efecto de la anestesia regional con la finalidad de reducir la resistencia muscular autónoma. Se realizaron las maniobras de cajón anterior y Pivot Shift usando el KT-1000 y los acelerómetros, respectivamente, en ambas rodillas. Posteriormente se procedió a realizar la reparación con técnica de Un Haz o la de «U-Dos» de forma aleatoria. La elección de la técnica se realizó de manera aleatorizada al obtener un sobre sellado de una urna, en el cual se encontró escrito Un Haz o U-Dos. Al finalizar el procedimiento quirúrgico se realizaron nuevamente las pruebas en la rodilla postoperada para valorar el estado de la misma.
Durante las citas de seguimiento del mes uno al tercero se le aplicó nuevamente a cada paciente ambos cuestionarios y se procedió a realizar las maniobras de cajón anterior mediante el KT-1000 y la de Pivot Shift utilizando los acelerómetros. Con los resultados obtenidos se realizó un análisis estadístico para obtener las integrales del eje de las X, Y y Z en las cifras que provee el Pivot Shift Meter, esto con el fin de disminuir los valores de interferencia que genera el simple movimiento de levantar la pierna para realizar la prueba; además, se obtuvieron las cifras de desplazamiento anterior que produce el KT-1000 y finalmente los resultados arrojados tras la realización de los cuestionarios de IKDC y Tegner Lysholm. Al utilizar dichos valores se realizó una comparación entre la progresión de los datos obtenidos para evaluar la mejoría de acuerdo a la evolución clínica y sintomatología del paciente.

Por último, se llevó a cabo un análisis estadístico con T-pareada para los promedios de los valores obtenidos por el artrómetro KT-1000 para cada una de las técnicas en diferentes tiempos.

Tabla 1: Valores obtenidos con KT-1000.

\begin{tabular}{|c|c|c|c|c|c|c|}
\hline \multirow{2}{*}{$\begin{array}{c}\text { Paciente } \\
\text { P1 }\end{array}$} & \multicolumn{2}{|c|}{ KT-1000 pre-Qx } & \multirow{2}{*}{$\begin{array}{c}\text { Rodilla sana } \\
3\end{array}$} & \multirow{2}{*}{$\begin{array}{c}\text { Diferencia con } \\
\text { rodilla sana } \\
6\end{array}$} & \multicolumn{2}{|c|}{ KT-1000 post-Qx } \\
\hline & $2,4,9$ & 9 & & & $1,2,3$ & 3 \\
\hline P2 & $2,4,7$ & 7 & 4 & 3 & $3,6,9$ & 9 \\
\hline P3 & $2,4,7$ & 7 & 4 & 3 & $2,3,4$ & 4 \\
\hline P4 & $1,3,5$ & 5 & 3 & 2 & $1,3,4$ & 4 \\
\hline P5 & $2,6,8$ & 8 & 7 & 1 & $1,3,5$ & 5 \\
\hline P6 & $3,5,7$ & 7 & 4 & 3 & $1,3,4$ & 4 \\
\hline P7 & $1,7,11$ & 11 & 6 & 5 & $1,3,4$ & 4 \\
\hline P8 & $4,7,9$ & 9 & 6 & 3 & $1,3,5$ & 5 \\
\hline P9 & $1,4,6$ & 6 & 3 & 3 & $1,3,4$ & 4 \\
\hline P10 & $2,4,8$ & 8 & 4 & 4 & $1,3,5$ & 5 \\
\hline P12 & $1,4,7$ & 7 & 7 & 0 & $1,2,4$ & 4 \\
\hline P14 & $1,4,7$ & 7 & 4 & 3 & $1,2,4$ & 4 \\
\hline P15 & $3,4,6$ & 6 & 4 & 2 & $1,3,4$ & 4 \\
\hline P16 & $4,7,9$ & 9 & 4 & 5 & $1,3,5$ & 5 \\
\hline P17 & $5,8,10$ & 10 & 4 & 6 & $3,4,6$ & 6 \\
\hline P18 & $3,5,8$ & 8 & 5 & 3 & $1,2,3$ & 3 \\
\hline P19 & $5,8,10$ & 10 & 5 & 5 & $1,3,5$ & 5 \\
\hline P20 & $4,8,14$ & 14 & 5 & 9 & $1,1,2$ & 2 \\
\hline P21 & $1,2,3$ & 3 & 3 & 0 & $1,2,2$ & 2 \\
\hline P22 & $3,7,9$ & 9 & 5 & 4 & $1,3,4$ & 4 \\
\hline P23 & $3,5,8$ & 8 & 4 & 4 & $1,2,3$ & 3 \\
\hline P24 & $2,4,6$ & 6 & 2 & 4 & $1,1,1$ & 1 \\
\hline P25 & $3,5,6$ & 6 & 6 & 0 & $1,2,3$ & 3 \\
\hline P26 & $3,4,8$ & 8 & 4 & 4 & $1,3,4$ & 4 \\
\hline P27 & $1,2,3$ & 3 & 3 & 0 & $1,2,2$ & 2 \\
\hline P28 & $3,8,11$ & 11 & 8 & 3 & $1,4,6$ & 6 \\
\hline P29 & $4,8,10$ & 10 & 6 & 4 & $2,3,4$ & 4 \\
\hline Р30 & $5,8,11$ & 11 & 6 & 5 & $2,5,6$ & 6 \\
\hline P31 & $3,6,8$ & 8 & 5 & 3 & $2,3,4$ & 4 \\
\hline P32 & $4,7,8$ & 8 & 3 & 5 & $1,2,3$ & 3 \\
\hline P33 & $3,7,8$ & 8 & 6 & 2 & $2,5,6$ & 6 \\
\hline P34 & $2,4,6$ & 6 & 4 & 2 & $1,3,5$ & 5 \\
\hline P35 & $3,5,6$ & 6 & 4 & 2 & $1,2,3$ & 3 \\
\hline P36 & $4,7,9$ & 9 & 2 & 7 & $1,2,2$ & 2 \\
\hline
\end{tabular}


A)

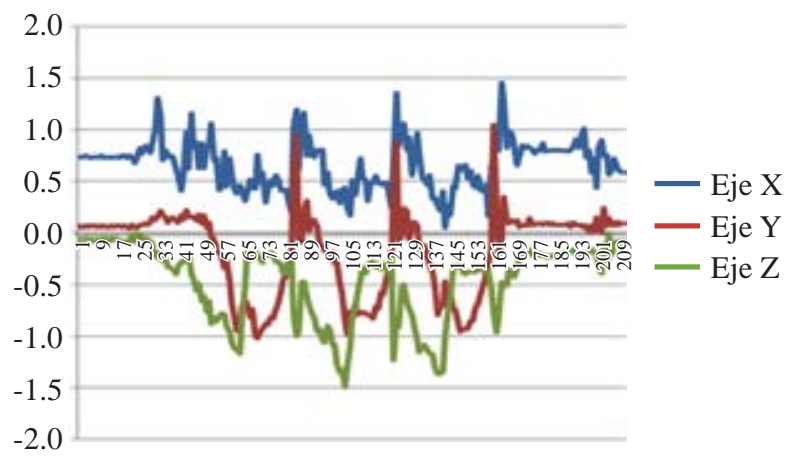

B)

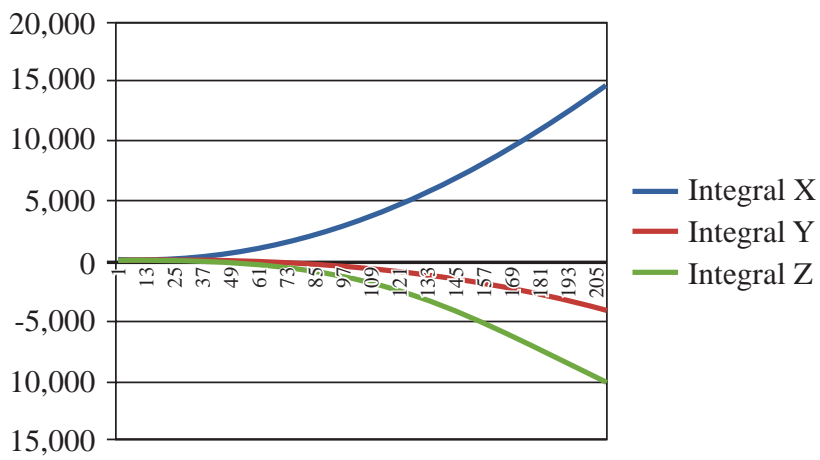

Figura 1: A) Gráfica obtenida con Pivot Shift Meter previo al cálculo de las integrales de cada eje de movimiento. B) Gráfica obtenida tras el cálculo de las integrales de cada eje de movimiento.

\section{Resultados}

De los 36 pacientes aleatorizados 18 (52.94\%) fueron sorteados para la técnica de Un Haz y 16 (47.06\%) para la de U-Dos. El total de la población contaba con diagnóstico de lesión de LCA por medio de pruebas clínicas y resonancia magnética (RMN) de la rodilla afectada.

Se excluyeron a dos pacientes del estudio debido a que fueron reintervenidos quirúrgicamente, debido a lesiones accidentales de la misma rodilla a la cual se le realizó la reconstrucción del LCA.

Para comparar la reparación de LCA entre las dos técnicas se capturaron los resultados obtenidos con el artrómetro KT-1000, ya que es el «estándar de oro» (Tabla 1). En el presente estudio $11.76 \%$ de los pacientes presentaron un desplazamiento mayor a $11 \mathrm{~mm}$ en la rodilla afectada, por lo que se consideraron como rotura completa del LCA; además, tres pacientes mostraron el mismo resultado en ambas rodillas tras la medición del cajón anterior, aun con diagnóstico confirmado por medio de RMN y posteriormente por artroscopía directa. Por lo tanto, se concluyó que un desplazamiento anteroposterior similar y una diferencia de 0 entre ambas rodillas del mismo paciente no descarta lesión de ligamento cruzado anterior, ya que el ligamento puede estar lesionado de forma parcial o incompleta, es decir, que la integridad del haz anterolateral (lesión parcial) puede evitar un desplazamiento evidente, medible por el artrómetro, dando un resultado falso negativo o en su defecto que el haz posterolateral sea el lesionado y no se detecte con KT-1000, a diferencia de las mediciones obtenidas por Pivot Shift Meter en una medición rotacional completa y no solamente anteroposterior.

Otro hallazgo importante en el estudio se relaciona con el paciente número 2. Dicho paciente presentó un desplazamiento anterior, medido por KT-1000, de $7 \mathrm{~mm}$ en la rodilla lesionada previo a la intervención quirúrgica y de $3 \mathrm{~mm}$ en la rodilla contralateral sana. Posterior a la cirugía el desplazamiento de la rodilla postoperada aumentó a $9 \mathrm{~mm}$, por lo que la diferencia entre ambas llegaría hasta los $6 \mathrm{~mm}$. Sin embargo, el paciente muestra una mejoría clínica significativa, ya que los valores obtenidos en los cuestionarios de
IKDC y la escala de Tegner Lysholm logran un aumento de 10 a 15 puntos en cada uno tras tres meses de seguimiento. Cabe mencionar que la técnica quirúrgica empleada en dicho paciente fue la de Un Haz.

Para comparar los resultados pre- y postoperatorios con el Pivot Shift Meter, se obtuvieron los valores de velocidad $(\mathrm{m} / \mathrm{s})$ generados con los acelerómetros, de los cuales se obtuvo la integral de cada eje de movimiento (X, Y y Z) para tomarlo como referencia en la pierna sana y en la pierna lesionada posterior al tratamiento quirúrgico, con la intención de determinar el grado de mejoría inmediata de la rodilla con lesión de LCA se tomó como referencia la pierna contralateral sana (Figura 1). Para ejemplificar los resultados obtenidos se tomaron gráficas de integrales obtenidas en pacientes con la mejoría más significativa tras artrometría con KT-1000. Los resultados mostraron diferencia significativa en el eje de las X, obteniendo un desplazamiento de hasta $\pm 4,000 \mathrm{~m} / \mathrm{s}$ en la estabilidad rotacional con la técnica de Un Haz (Figura 2). Se puede decir que se logró una recuperación parcial de la estabilidad anteroposterior, mas no rotacional. Por el otro lado, con la técnica de reconstrucción de U-Dos la diferencia de desplazamiento entre la pierna sana control y la pierna lesionada postoperada fue menor $\pm 600 \mathrm{~m} / \mathrm{s}$ (Figura 3).

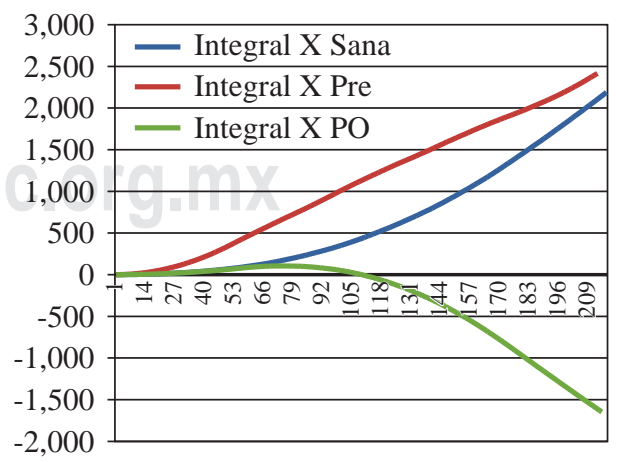

Figura 2: Integral del eje «X» en pacientes postoperados con técnica de reconstrucción de Un Haz, la cual muestra diferencia de velocidad en ACM de $\pm 4,000 \mathrm{~m} / \mathrm{s}$ entre pierna sana y pierna PO (postoperada). 


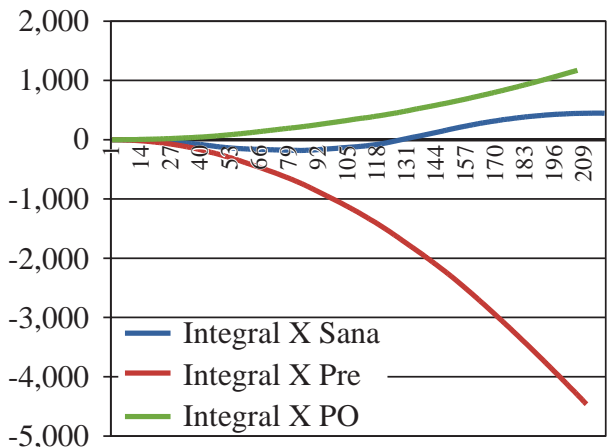

Figura 3: Integral del eje «X» en pacientes postoperados con técnica de reconstrucción de U-Dos, la cual muestra diferencia de velocidad en ACM de $\pm 600 \mathrm{~m} / \mathrm{s}$ entre pierna sana y pierna PO (postoperada).

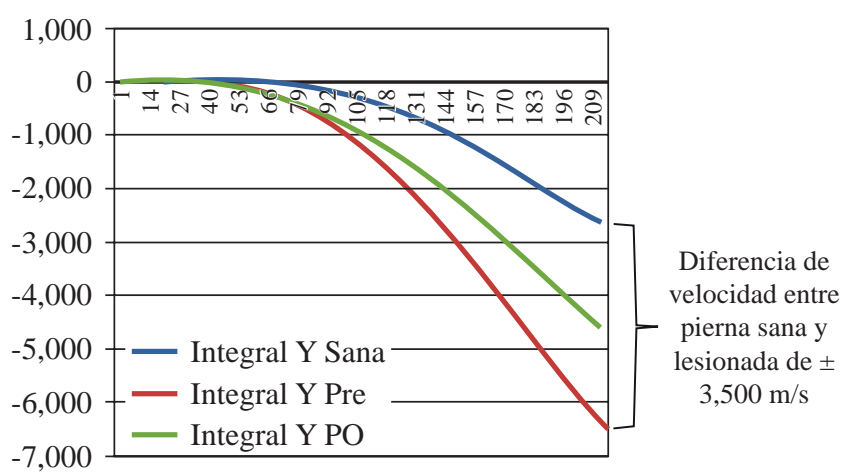

Figura 4: Ejemplo de un paciente cuya integral del eje «Y» muestra diferencia de velocidad en ACM de $\pm 3,500 \mathrm{~m} / \mathrm{s}$ entre la pierna sana y la lesionada en comparación con la PO que es menor a 2,500 m/s. Técnica de reconstrucción de U-Dos.

En cuanto a los valores de $\mathrm{Y}$, tenemos que la integral obtenida al medir la pierna sana fue de $-4,149.43 \mathrm{~m} / \mathrm{s}$, contra la rodilla lesionada previo a la anestesia que resultó en $-6,528.78 \mathrm{~m} / \mathrm{s}$. Posteriormente se obtuvo un valor de $-6,973.85 \mathrm{~m} / \mathrm{s}$ tras la reconstrucción del LCA mediante la técnica de Un Haz (Figura 4). En la integral de Y en un paciente con reconstrucción mediante U-Dos, obtuvimos $-2,760.09 \mathrm{~m} / \mathrm{s}$ en la rodilla sana y $-6,470.80 \mathrm{~m} / \mathrm{s}$ en el preoperatorio, mientras que en el postoperatorio se obtuvo $-4,670.07 \mathrm{~m} / \mathrm{s}$ (Figura 5).

Para los valores del eje Z se obtuvieron los siguientes valores: la rodilla sana obtuvo un valor de $-893.27 \mathrm{~m} / \mathrm{s}$, la rodilla afectada obtuvo $-3,964$. en el prequirúrgico y finalmente $-2,276.6 \mathrm{~m} / \mathrm{s}$ en el postquirúrgico tras la reconstrucción con Un Haz (Figura 6). Mientras que con U-Dos resultaron en -732.5 para la rodilla sana, $-5,517.06$ para la rodilla afectada previo a la cirugía y $-3,510.08$ posterior a la misma (Figura 7).

Se dio seguimiento a los 36 pacientes mediante las escalas de valoración clínica que se aplicaron antes de la cirugía y a diferentes tiempos postoperatorios para cada grupo de pacientes operados con las dos técnicas de reconstrucción. Se obtuvieron las medias \pm desviación estándar de los pacientes operados con la técnica de Un Haz: los resultados preoperatorios para la escala Tegner Lysholm fueron de $57 \pm 6.64$ puntos, mejorando a los tres meses posteriores (65 \pm 11.4 puntos), pero no de manera significativa. Con la escala de IKDC los valores preoperatorios fueron de $43.7 \pm 12.1$ puntos, comparados a $46 \pm 6.68$ puntos después de tres meses después a la cirugía, de igual manera, sin diferencia significativa. Los resultados de las mismas escalas para la técnica de reconstrucción de U-Dos fueron: para Tegner Lysholm de $57 \pm 13.7$ puntos en el preoperatorio y de $89 \pm 5.68$ puntos en la visita postoperatoria de tres meses, siendo la mejoría estadísticamente significativa $(\mathrm{p} \leq 0.05)$. De igual forma, con la escala IKDC con un puntaje de $41.4 \pm 17.7$ en el preoperatorio contra $84 \pm 15$ puntos a los tres meses posteriores $(p=0.001)$.

El seguimiento de los pacientes también incluyó mediciones con KT-1000 y los valores que se obtuvieron con la técnica de U-Dos fueron estadísticamente superior a los de Un Haz ( $p=0.002)$. La importancia radica en el nivel de recuperación de la estabilidad completa para la técnica U-Dos con una disminución del valor promedio inicial prequirúrgico de $2.8 \mathrm{~mm}$ hasta $-0.9 \mathrm{~mm}$ para la primera visita al mes y de igual manera de $-0.9 \mathrm{~mm}$ para la segunda visita a los tres meses, todo esto a diferencia de los valores obtenidos para la técnica de Un Haz con una estabilidad parcial, reduciendo

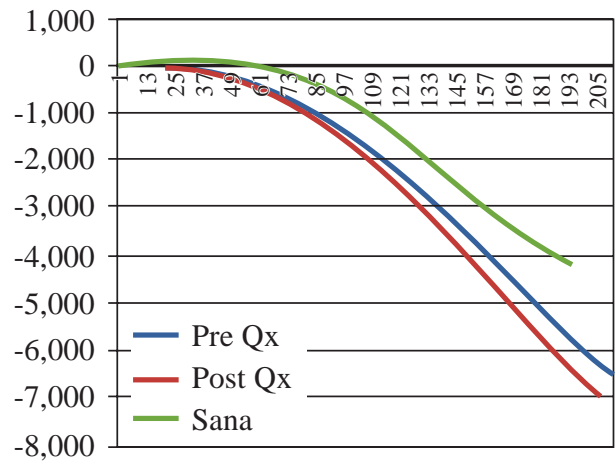

Figura 5: Integrales del eje «Y» obtenidas en un paciente PO bajo técnica de Un Haz. Se observa una similitud entre las líneas pre- y post Qs; sin embargo, existe una mayor distancia entre la sana y la postoperada.

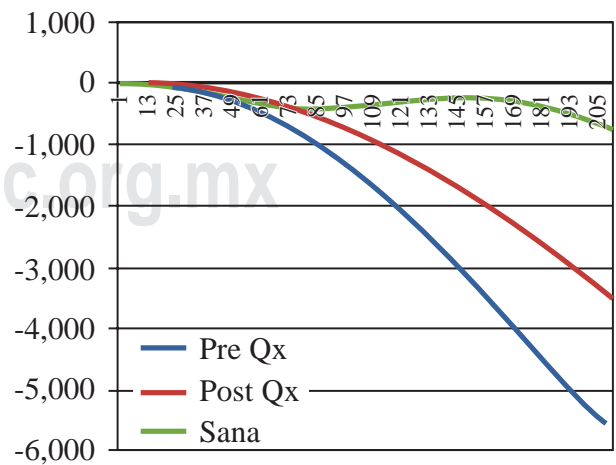

Figura 6: Integral del eje «Z» en pacientes postoperados con técnica de reconstrucción de Un Haz, la cual muestra diferencia de velocidad en ACM de $\pm<2,700 \mathrm{~m} / \mathrm{s}$ entre pierna sana y pierna PO (postoperada). 


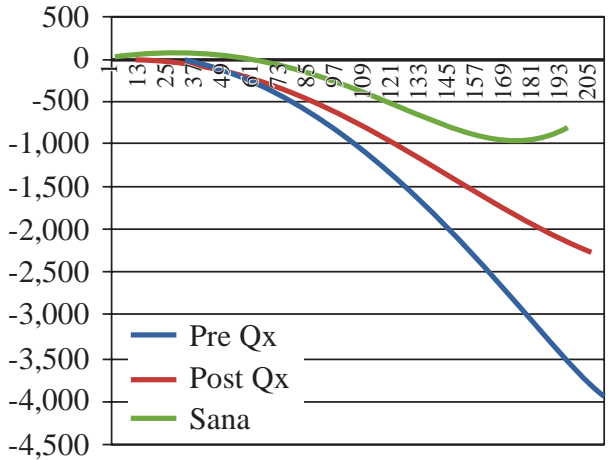

Figura 7: Integral del eje «Z» en pacientes postoperados con técnica de reconstrucción de U-Dos, la cual muestra diferencia de velocidad en ACM de $\pm 2,500 \mathrm{~m} / \mathrm{s}$ entre pierna sana y pierna postoperada.

los valores promedio de $3.5 \mathrm{~mm}$ hasta $0.2 \mathrm{~mm}$ en la segunda visita a los tres meses.

\section{Discusión y conclusiones}

En este estudio se logró medir la aceleración en los tres ejes X, Y, Z mediante el Pivot Shift Meter. Las aceleraciones de los ejes $\mathrm{Y}$, X se transformaron a $\mathrm{m} / \mathrm{s}$, unidad de velocidad, calculando la integral de cada eje. Se tomó como base la hipótesis de que entre mayor similitud haya entre las gráficas obtenidas en el postoperatorio y la gráfica de la rodilla sana, mayor es la efectividad de la técnica empleada, ya que el valor de referencia que se toma como «normal» sería la rodilla contralateral o «sana» del paciente. Se compararon los resultados de Pivot Shift Meter entre las técnicas de Un Haz y la de U-Dos, demostrando en diversos pacientes mejores resultados con esta última técnica al comparar resultados prey postoperatorios. Se comparó la evolución de los pacientes entre las dos técnicas de reparación mediante KT-1000, escalas Tegner Lysholm e IKDC mostrando mejoría significativa a corto plazo (tres meses) con la técnica de U-Dos.

En cuanto a modificaciones a futuro encontramos que es necesario una mayor difusión de la técnica correcta para realizar la maniobra del Pivot Shift en nuestro grupo de trabajo y lograr la subluxación en pacientes cuyo LCA se encuentre comprometido, ya que las variaciones en cuanto a la ejecución de la misma, incluso en las manos de un mismo examinador, podría llevar a la detección de falsos positivos o negativos, disminuyendo la sensibilidad de la maniobra de manera considerable. Además, se sugirió la creación de un video tutorial o diagrama de flujo que acompañe a la aplicación o al equipo para la medición, con instrucciones precisas para la realización de cada paso de la maniobra: velocidad, fuerza y cantidad de repeticiones dentro de límites determinados, para una mayor precisión en la obtención de datos y mayor sensibilidad y especificidad.

Algunos cambios que se buscan a futuro son la mejoría y actualización del equipo tanto en el hardware como en el software para la recolección de datos y valoración de los mismos, ya que existen diferentes «bugs» o errores en el sistema que llevan a la pérdida de información y corrupción de los archivos generados. Esto ocasiona que el sistema deba reiniciarse y se realicen múltiples veces la maniobra para asegurarse de la correcta captura de los datos a evaluar.

Consideramos que aún es necesaria la valoración a largo plazo de los pacientes postoperados de reparación de LCA con ambas técnicas para evaluar la evolución tras seis meses a dos años de la cirugía como mínimo. Esto generará más datos que lleven a una conclusión para definir las diferencias obtenidas mediante las diferentes técnicas y comprobar si éstas son suficientes como para una selección más precisa de la misma. Nuestra muestra es pequeña por lo que la validez y la veracidad de los datos obtenidos aún son cuestionables.

Bibliografía

1. Hensler D, Van Eck CF, Fu FH, Irrgang JJ. Anatomic anterior cruciate ligament reconstruction utilizing the double-bundle technique. $J$ Orthop Sports Phys Ther. 2012; 42(3): 184-95.

2. Brucker P, Imhoff A. Aperture fixation in primary arthoscopic double-bundle, single-bundle, and single-bundle-augmented ACL Reconstruction. Freddie H Fu. MD. Sports Medicine, Master Techniques in Orthopaedic Surgery. Chapter 29, Lippincott Williams \& Wilkins; 2010, pp. 235-44.

3. Li X, Xu C, Song J, Jiang N, Yu B. Single-bundle versus doublebundle anterior cruciate ligament reconstruction: an up-to-date metaanalysis. Int Orthop. 2013; 37(2): 213-26.

4. Tashman S, Collon D, Anderson K, Kolowich P, Anderst W. Abnormal rotational knee motion during running after anterior cruciate ligament reconstruction. Am J Sports Med. 2004; 32(4): 975-83.

5. Adachi N, Ochi M, Uchio Y, Iwasa J, Kuriwaka M, Ito Y. Reconstruction of the anterior cruciate ligament. Bone Jt J. 2004; 86(4): 515-20.

6. Lee BI, Kwon SW, Choi HS, Chun DI, Kim YB, Kim BM. Anatomic single-bundle anterior cruciate ligament reconstruction with remnant preservation using outside-in technique. Arthrosc Tech. 2015; 4(4): e331-4.

7. Meredick RB, Vance KJ, Appleby D, Lubowitz JH. Outcome of single-bundle versus double-bundle reconstruction of the anterior cruciate ligament: a meta-analysis. Am J Sports Med. 2008; 36(7): 1414-21.

8. Berumen-Nafarrate E, Leal-Contreras C. Double-bundle and doubletunnel ACL reconstruction with looped proximal tibial fixation. Orthopedics. 2011; 34(6): 441.

9. Morales C, Paz M, Berumen E, Leal I, Leal C. Plastía de ligamento cruzado anterior con técnica de "U-Dos”. Acta Ortopédica Mex. 2013; 27(3): 142-8.

Financiamiento: Todos los gastos incurridos al realizarse el estudio fueron absorbidos por los autores del artículo. 